\title{
Encephalopathy in a case of Whipple's disease
}

\author{
J. BADENOCH, W. C. D. RICHARDS, AND D. R. OPPENHEIMER \\ From the Nuffield Department of Clinical Medicine and the Departments of \\ Pathology and Neuropathology, Radcliffe Infirmary, Oxford
}

In 1907, Whipple described the case history and post-mortem findings in a young physician who in life had suffered from cough, loss of weight, arthralgia, and diarrhoea. At necropsy deposits of fat were found in the intestinal mucosa and in the mesenteric lymph nodes. These appearances led Whipple to suggest intestinal lipodystrophy as a name for the disease (Whipple, 1907). Cases of Whipple's disease are rare, but there are now over 70 described in the world's literature. It has been established that a characteristic feature of the disease is the presence in the intestinal mucosa of macrophages containing a glycoprotein (Casselman, Macrae, and Simmons, 1954) and that lesions containing similar macrophages may be found in many tissues of the body. In this connexion it is of interest that Whipple himself mentioned the presence of non-lipid foam cells in the intestinal mucosa and mesenteric glands. This paper deals with a case of Whipple's disease in which cerebral lesions were found at necropsy.

Attention was first drawn to involvement of the central nervous system by Sieracki, Fine, Horn, and Bebin in 1960, who reported pathological changes in the substance of the brain in two cases, both male, and neither of which had had any neurological symptoms during life. However, in January 1962 Lampert, Tom, and Cumings reported a further patient who had never suffered from diarrhoea, whose illness was characterized by a disturbance of behaviour, and who died seven years after the onset after a short period of drowsiness and stupor. On examination towards the end of his life he was found to have divergent strabismus, with paresis of conjugate gaze laterally and upwards, clonic movements of the left side of the face and the left arm, and vertical nystagmus. The cerebrospinal fluid contained $18 \mathrm{mg}$. of protein per $100 \mathrm{ml}$. and its pressure was normal. Bilateral angiograms did not reveal any abnormality, but an air encephalogram showed the brain to be atrophied. At necropsy the typical appearances of Whipple's disease were found in the mesenteric lymph nodes and in the submucosa of the jejunum, and widespread changes were present in the grey matter of the brain.
These authors regard the involvement of the nervous system in their cases as a simple, though unusual, extension of the primary disease process. In the case which we are about to report, which has many features in common with that of Lampert et al. (1962), we believe that there is evidence of a secondary factor, namely, a Wernicke type of encephalopathy, attributable to a chronic state of malnutrition.

The patient was a seed merchant who was first admitted to the Radcliffe Infirmary in November 1956 at the age of 48, with a history of loss of weight for two years, and painless, bulky diarrhoea without obvious blood or mucus in the stools for 18 months. Examination revealed that he was pale and wasted with a low-grade fever. The abdomen was soft and not tender and no viscera or abnormal masses could be felt. Investigation showed a moderate iron-deficiency anaemia, with a haemoglobin of $10 \mathrm{~g}$. per $100 \mathrm{ml}$. and severe steatorrhoea, the daily faecal excretion of fat being $28 \mathrm{~g}$. At first he was thought to be suffering from idiopathic steatorrhoea, but the raised E.S.R., the presence of occult blood in the stools, and the demonstration that radioactive iron could be absorbed normally all suggested that the steatorrhoea was secondary to an anatomical lesion of the bowel. A laparotomy was performed and typical changes of Whipple's disease were found. A biopsy was obtained from the jejunal mucosa and a mesenteric lymph node was taken for histological study. The specimen consisted of a piece of jejunum $2 \times 1 \times 0.5 \mathrm{~cm}$. and a mesenteric lymph node $1 \times 0.5 \mathrm{~cm}$. The mucosal aspect of the jejunum presented a coarse yellowish granularity.

The histological appearances were typical of Whipple's disease. The lamina propria of the jejunal mucosa was packed with large mononuclear cells which had a granular or foamy cytoplasm. Rounded clear spaces were present, mainly in the middle and lower parts of the mucosa (Fig. 1). Staining by the periodic-acid-Schiff technique showed the cytoplasm of the mononuclear cells to contain P.A.S.-positive material in diffuse and granular form (Fig. 2). Sudanophilic lipid was present in the clear spaces and there was much finely divided lipid in the superficial parts of the lamina propria. The mucosal infiltrate had resulted in separation of the gland tubules and caused gross distortion of the intestinal villi.

Sections of the mesenteric lymph node showed a honeycomb structure due to dilatation of the sinuses and P.A.S.-positive macrophages were present in the 


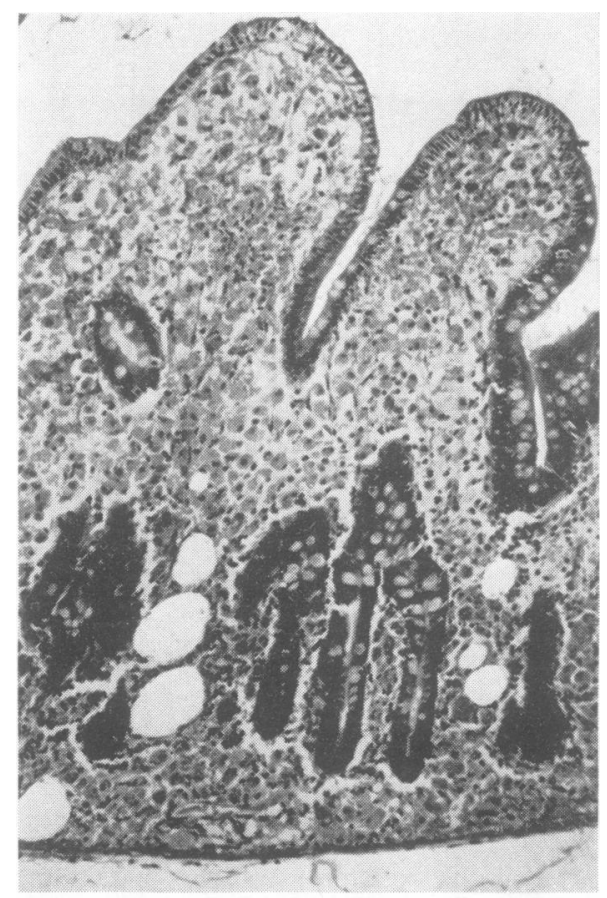

FIG. 1

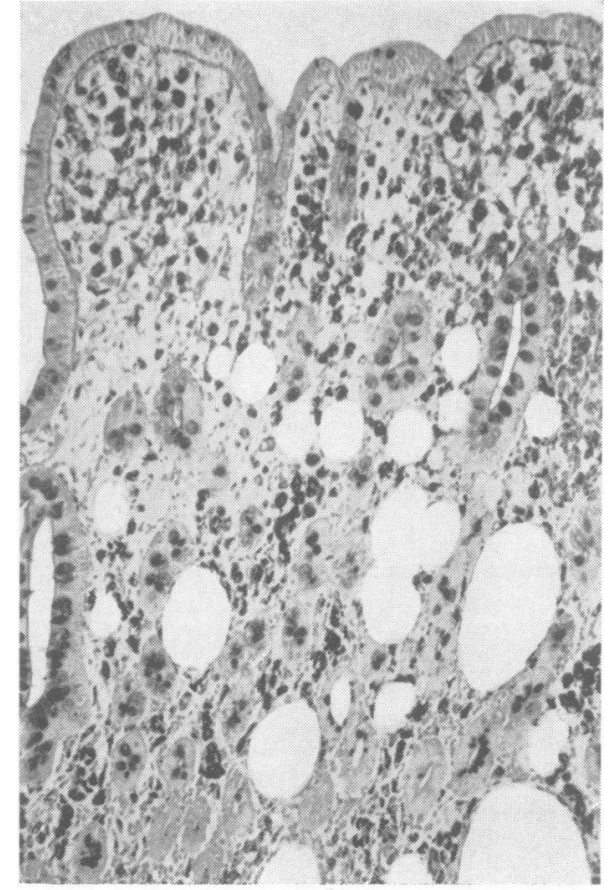

FIG. 1. Jejunal mucosa showing fat spaces and the characteristic cellular infiltrate of Whipple's disease (haematoxylin and eosin $\times 95$ ).

FIG. 2. Jejunal mucosa showing P.A.S.-positive material in the mononuclear cells (P.A.S. $\times$ 95). lymphoid tissue either singly or in small aggregates, which sometimes resembled epithelioid follicles.

Over the next three years his illness pursued a stormy course. Steroids failed to bring about improvement, and although $x$ rays to the abdomen seemed to produce benefit for a time, he gradually went downhill. There were episodes of severe hypokalaemia and subacute intestinal obstruction. He was put on a régime which included full vitamin and mineral supplements, including whole B complex in large doses, but he took his treatment irregularly and gave it up for long periods at a time. In the later stages of his illness his increasing apathy and malnutrition made management difficult.

In April 1959, four and a half years after the onset of his illness, the patient returned to hospital with blurred vision, diplopia, and muzziness in the head. On examination he was alert and responsive but disoriented and had amnesia for recent events; the pupils were equal and regular, but did not react to light and there was minimal spontaneous nystagmus at rest and a gaze paralysis which fluctuated from week to week. He had a slight dysarthria, and there was mild ataxia, but no other abnormalities could be found in the central nervous system. Vigorous treatment with full supplements of vitamins and minerals and prednisone, in a dose of $20 \mathrm{mg}$. three times a day, improved his general condition to some extent for a time, but during the last two months of his life he became less and less interested in his surroundings. He lay in bed with his upper eyelids drooping over his motionless eyes, and remained in a state of semi-stupor from which he could be aroused only by loud questioning. A few days before the end he lapsed into a coma and he died on 2 Novembe 1959 , five years after the onset of his illness.

The cerebrospinal fluid was examined in April 1959 at the onset of neurological symptoms. It was clear and colourless with a pressure of $100 \mathrm{~mm}$. of cerebrospinal fluid. The protein was $33 \mathrm{mg}$. per $100 \mathrm{ml}$. and there were 5 cells, 1 polymorph, and 4 lymphocytes per c.mm. The Lange curve was normal and the Wassermann reaction negative. The electroencephalogram showed a mildly unstable $9 \mathrm{c} / \mathrm{s}$ alpha rhythm, mixed with $16 \mathrm{c} / \mathrm{s}$ beta activity. The record was considered to be normal, and there was no focus to suggest a cerebral tumour. Air ventriculography and air encephalography were carried out, but no space-occupying lesion was found within the skull.

The electroencephalogram was repeated towards the end of his life, and it showed gross widespread and diffuse slowing and suppression of cerebral rhythm.

\section{POST-MORTEM EXAMINATION}

Externally the body was that of a wasted middleaged man with grey hair.

THORAX A little blood-stained fluid was found in both pleural cavities and there were fibrous adhesions between the posterior parts of the lower lobes of the lungs and the parietal pleura. The lungs (R.756 g., L.620 g.) showed oedema and tubular bronchiectasis of the lower lobes. The 
bronchi contained mucopus and were lined by hyperaemic mucosa. The heart (378 g.) was of normal size and shape. The visceral and parietal layers of pericardium were firmly stuck together by soft fibrous adhesions. Scattered small white flecks were present on the visceral pericardium. No abnormality was seen in the myocardium. On the auricular surface of the mitral valve tiny tan-coloured vegetations were visible just above the free margin. Similar vegetations were seen on the aortic valve and its commissures. The coronary arteries were normal, and no abnormality was found in the aorta and its branches.

ABDOMEN The liver $(1,628 \mathrm{~g}$.), the spleen $(116 \mathrm{~g}$.), and the pancreas were normal. The gall bladder contained a solitary mixed pigment and cholesterol stone. The small intestine was dilated and except for the terminal ileum presented a dark brown appearance. The mucosal folds were well preserved in the jejunum, but not elsewhere. A faint yellowish granularity was observed in the jejunal mucosa but the appearances were obscured by advanced postmortem autolysis. Apart from gaseous distension, the large bowel was normal. The mesentery contained a number of slightly enlarged lymph nodes which measured up to $1.5 \mathrm{~cm}$. in diameter. The cut surfaces of these nodes presented a mottled grey and red appearance without evidence of cystic or honeycomb structure. Lipid deposits were not visible to the naked eye and the mesenteric lymphatics were not dilated.

\section{GENITO-URINARY SYSTEM This was normal.}

LYMPH NODES There was no significant enlargement of the peripheral groups of lymph nodes.

BONES AND JOINTS Deep red marrow was present in the sternum and vertebral bodies. The femoral marrow was fatty with a few greyish-pink areas. There was no evidence of osteomalacia or osteoporosis. No lipid deposits were seen in the synovial membranes. The left knee joint showed osteoarthritis.

\section{ENDOCRINE GLANDS These were normal.}

HISTOLOGY The jejunal mucosa and mesenteric lymph nodes presented essentially similar appearances to those described in the biopsy specimens. The detailed mucosal structure was obscured by post-mortem changes. Lipofuscin pigment was found in the muscular layer of the small bowel. Fat was present in the dilated sinuses of the mesenteric lymph nodes.
The aortic and mitral valve vegetations consisted of fibrous tissue capped by deposits of fibrin in which P.A.S.-positive macrophages were found. Small collections of P.A.S.-positive macrophages were found in the axillary, cervical, and inguinal lymph nodes, in the pericardium, and in one small myocardial artery.

No lesions of Whipple's disease were found in the skin, tongue, submandibular gland, liver, kidney, spleen, prostate, testis, lung, bone marrow, pancreas, thyroid, adrenal, and pituitary. There was a chronic osteoarthritis of the knee joint. No P.A.S.-positive cells were found in the synovia of the knee, shoulder, and elbow joints.

\section{CENTRAL NERVOUS SYSTEM}

The brain (1,520 g.) appeared normal externally, and there was no sign of vascular disease. Coronal slices through the cerebrum after fixation showed a moderate dilatation of the lateral and third ventricles, but no sign of obstructive hydrocephalus, and no obvious cortical atrophy. The ependyma looked normal. The only visible cerebral lesion was a small area of spongy degeneration in the body of the right caudate nucleus. A similar lesion was found in the anterior medullary velum. There were some dilated veins in the cerebellar white matter. The ependyma of the fourth ventricle showed a finely granular surface. Otherwise the brain-stem and cerebellum appeared normal.

HISTOLOGY Blocks, including frontal, temporal, parietal and occipital cortex and white matter, thalamus, hypothalamus and basal ganglia (four levels), midbrain (two levels), pons, medulla, cerebellum, and spinal cord (three levels), were embedded in paraffin and stained for cells, myelin, and fibroglia. Periodic-acid-Schiff staining was carried out on all these, along with normal and pathological controls. In addition, frozen sections of thalamus were stained for axons, neuroglia, microglia, and fat.

Microscopic lesions of four types were observed. The first, and most widespread, consisted of innumerable tiny deposits of an abnormal P.A.S.positive material. Each lesion was roughly circular, with a diameter of 0.1 to $0.2 \mathrm{~mm}$., and under higher magnifications (Figs. 3a and b) was seen to consist of a diffuse, finely granular dust of P.A.S.-positive material. Most, but not all, of the lesions included a few microglial phagocytes, containing material of the same kind, but more deeply stained and presumably in a more condensed state. Apart from this, there was no cellular reaction to the abnormal substance; and the substance itself, where it was not 


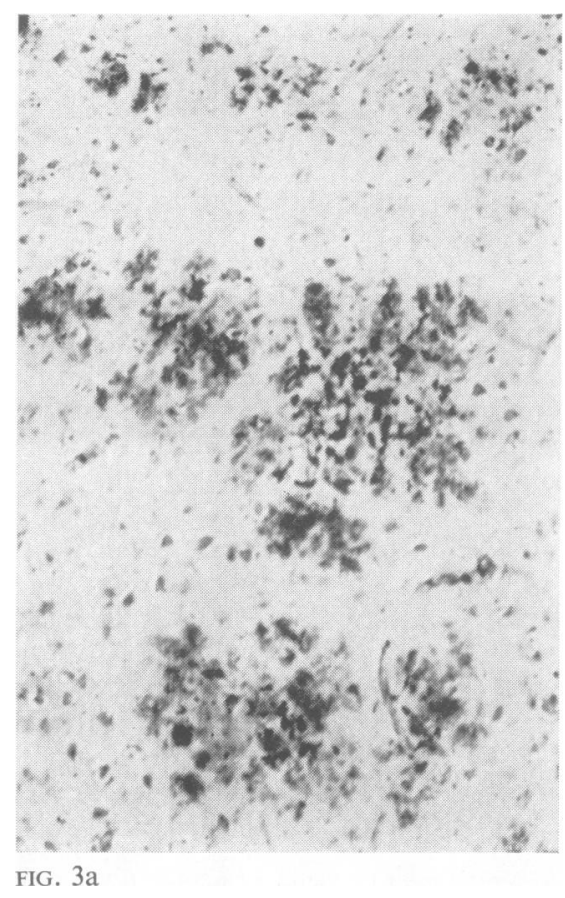

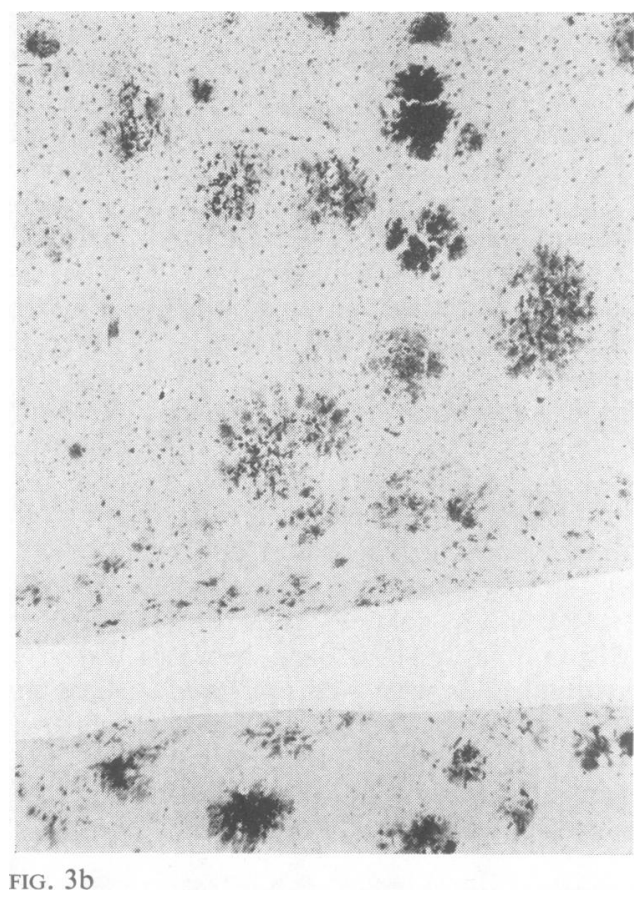

FIG. $3 \mathrm{a}$ Amygdaloid nucleus, showing lesions of first type. Some of the material is contained in phagocytes (stained dark); most of it appears to be extracellular (P.A.S. $\times$ 95).

FIG. $3 b$

Similar lesions in temporal cortex (P.A.S. $\times$ 38). contained in phagocytes, appeared to be entirely extracellular (whether it was truly extracellular, or contained in the ramifying processes of glial cells, could only be determined by means of electron microscopy). The site of the lesions appeared to be quite independent of blood vessels of capillary or precapillary size, an indication that the abnormal material had not reached the tissue by simple diffusion from the blood. None of the material was seen in the bodies of neurones or glial cells. Lipochrome, which stains positively with P.A.S., was present in normal amounts in large neurones in the thalamus and elsewhere; in haematoxylin-andeosin-stained preparations, this showed as brown globules, whereas the abnormal substance was either invisible or pale grey. It was noted that the substance was strongly argyrophilic in both paraffin and frozen sections, and stained dark blue in Holzer preparations.

In general, lesions of this type were found throughout the grey matter of the brain and spinal cord and not in the white matter. There were exceptions to this rule; for instance, the anterior pillars of the fornix and the white matter of the cerebellum contained lesions and the grey matter of the lower part of the spinal cord did not. For the rest, wherever there were nerve cells, either in compact nuclei or scattered among myelinated fibre tracts, there were lesions; and in large sections, stained by the P.A.S. method, the grey matter was clearly distinguishable by a fine red stippling (Fig. 4). This stippling varied somewhat in density. For instance, the thalamuso showed a greater concentration of lesions in itso medial than in its lateral parts, the striatum was more affected than the pallidum, and there were variations in different areas of cerebral cortex.

The second type of lesion consisted of a great proliferation of both microglia and astrocytes, with fibrillar gliosis. Associated with this was a patchy 'cuffing' of vessels of precapillary size with phagocytic cells. Both these and the microglia were densely packed with P.A.S.-positive material, having the same staining properties as the material in lesions of the first type (Fig. 5). Changes of the second type were seen in a few well-defined areas, including the fornix, the anterior and medial nuclei of the thalamus, the mammillary bodies, the supraoptic and paraventricular nuclei, the amygdaloid nucleus, and the hippocampus. The most severely affected area was the infundibular region of the hypothalamus, where proliferation of cells and capillaries reached an almost tumour-like density (Fig. 6).

Lesions of a third type were seen in the walls of the ventricles, especially the fourth ventricle, and the aqueduct. These consisted of small ependymal granulations, not different in kind from those met with in 'normal' brains, but unusually widespread and florid (Fig. 7). In addition, the free surface of the ependyma in most areas was coated with a fine 

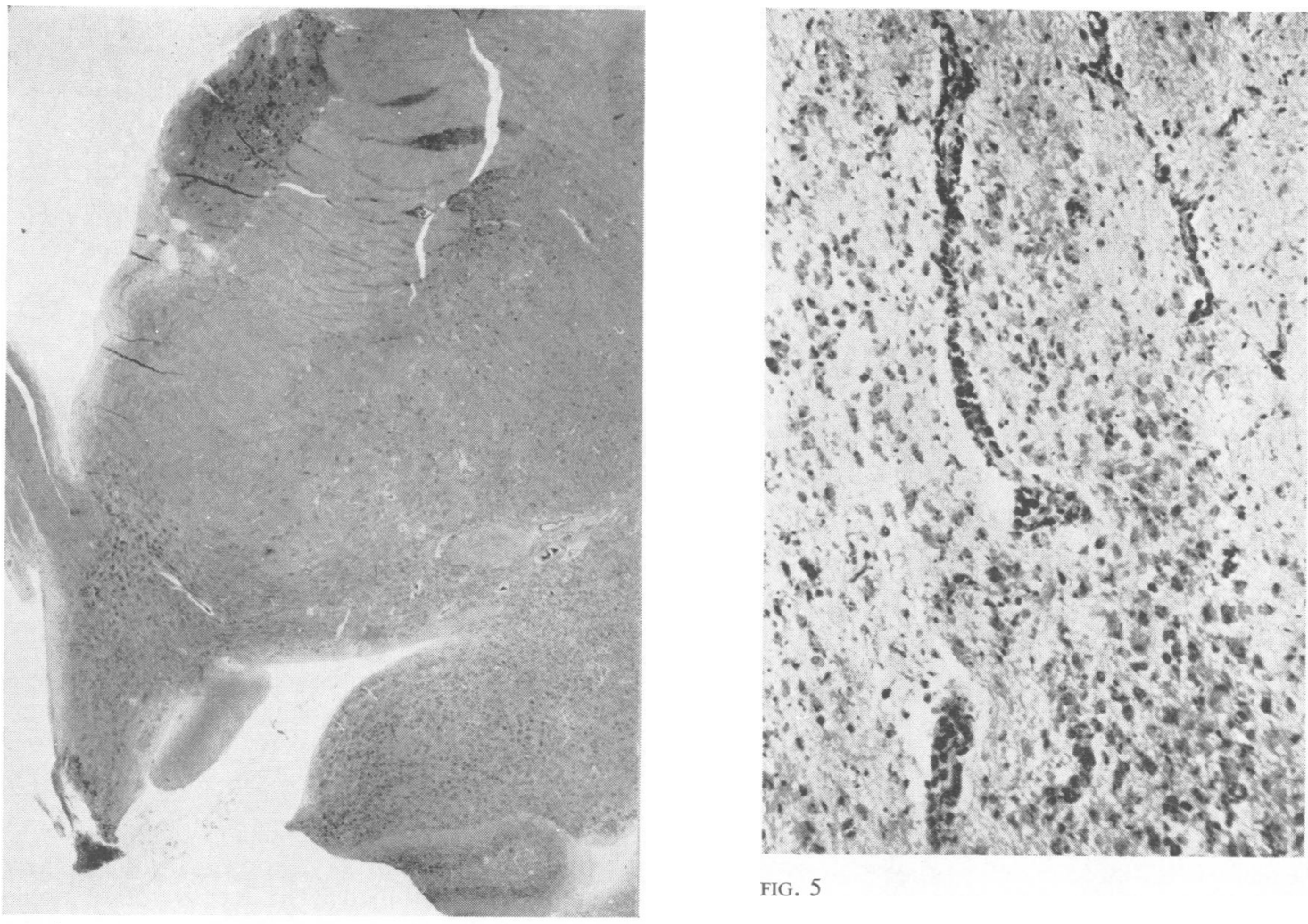

FIG. 5

FIG. 4

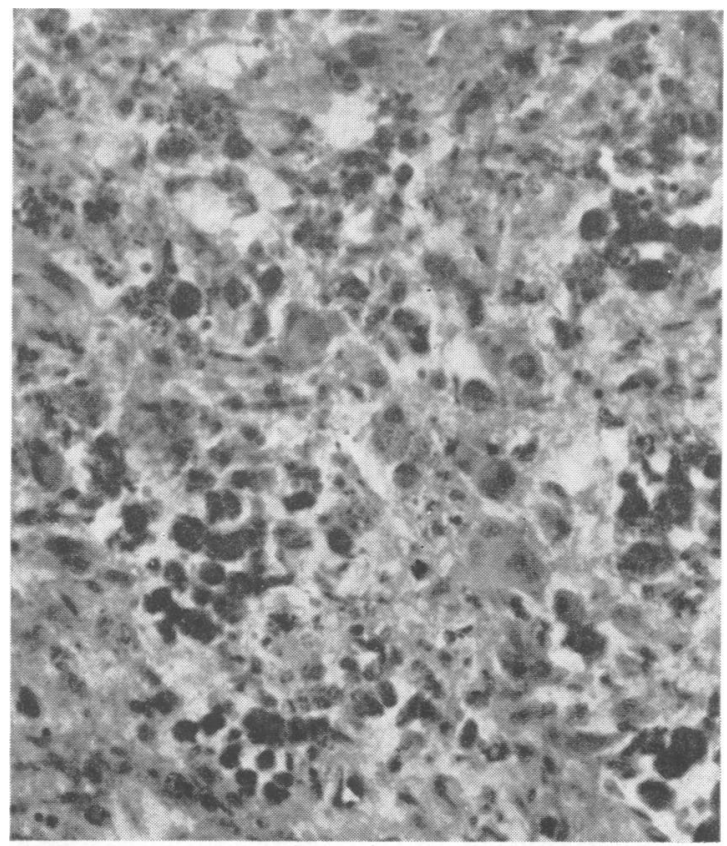

FIG. 4. Hypothalamus and basal ganglia in coronal section. Note that the material is confined to grey matter and the anterior pillar of the fornix. The internal capsule, optic tract, and anterior commissure are free (P.A.S. $\times 2 \cdot 5)$.

FIG. 5. Tuber cinereum, showing lesions of second type. Note cellular proliferation and accumulation of perivascular phagocytes (haematoxylin and eosin $\times 95$ ).

FIG. 6. Tuber cinereum. Area of intense proliferation of astrocytes and microglial phagocytes. Nerve cells are present (haematoxylin and eosin $\times 245$ ).

FIG. 6 


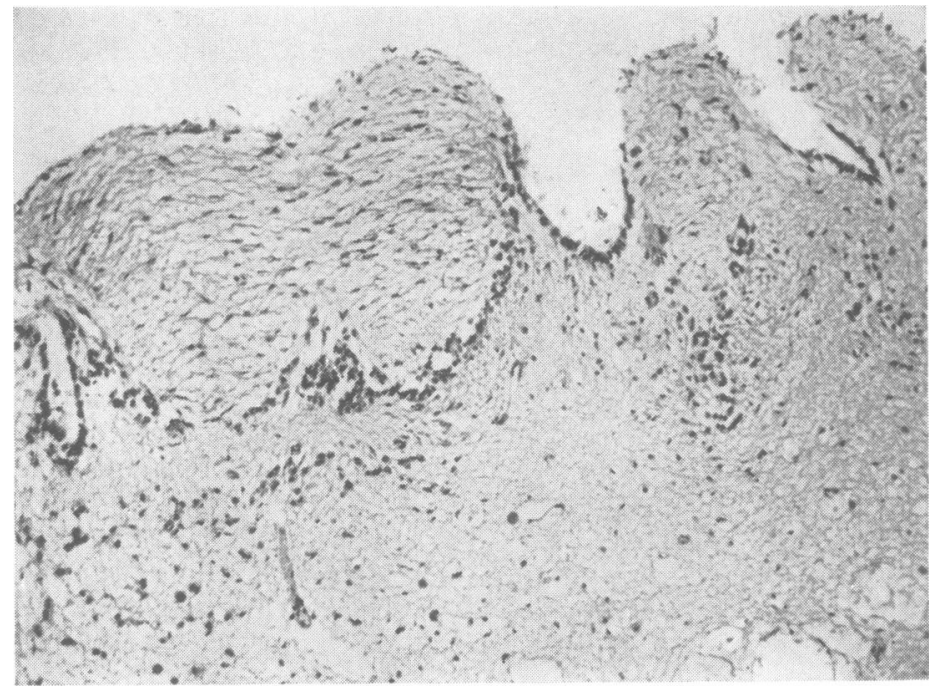

FIG. 7. Ependymal 'granulations' in aqueduct (haematoxylin and eosin $\times$ 95).

deposit of P.A.S.-positive granules. This was also seen on the epithelium of the choroid plexus, suggesting the presence of an abnormal chemical constituent in the cerebrospinal fluid. The ependymal granulations contained scattered phagocytic cells with P.A.S.-positive contents.

A fourth type of lesion was seen in two places, referred to in the naked-eye description, namely, the body of the right caudate nucleus and the anterior medullary velum. In the former, the tissue was spongy and degenerate, without lipid phagocytosis and with only mild gliosis. The veins in this region showed hyaline thickening. The anterior medullary velum was similarly affected; myelinated fibres of the trochlear decussation were seen passing through it, but were possibly reduced in number.

On the negative side, it is worth remarking that structural changes in nerve cells, or reductions in their numbers, were not observed in any part of the nervous system, with the possible exception of the hypothalamus, where proliferation of other tissues made judgment difficult. Nor was there any sign of damage to nerve fibres or myelin sheaths, except in the ansa lenticularis, which was gliotic and lacking in myelin. Finally, no sudanophilic lipid was seen, either free or within cells.

In view of the patient's most striking neurological peculiarity, paralysis of gaze, it is especially worth noting that no specific lesions (other than those of the first type, which were universal throughout the grey matter) were found in the oculomotor nuclei or tectum, or in the dorsal longitudinal bundle. The rather doubtful lesion of the trochlear decussation would not, in any case, account adequately for this symptom.
Peripheral nerve and muscle Sections of sciatic nerve showed no abnormalities except for slight interstitial fibrosis and round cell infiltration. Sections of muscle from upper and lower limbs showed atrophy of scattered muscle fibres, and silver impregnations on frozen sections revealed degenera- $\triangle$ tive fragmentation of many axons, indicating a fairly severe motor neuropathy. No deposits of 'Whipple substance' were seen in either nerve or muscle. Tangential and vertical frozen sections of skin, impregnated for nerve fibres, showed numerous axons undergoing fragmentation.

Occasional isolated fine terminal fibres showed fusiform swellings and terminal knobs, appearances generally associated with regenerative growth.

To summarize the pathological findings, the intestine showed the characteristic picture of Whipple's disease, and there were changes in lymph nodes and endocardium such as have been described in previous reports (Farnan, 1958). In addition, the grey matter of the brain and spinal cord showed a universal spattering with a P.A.S.-positive material, which appears to be identical with the 'Whipple substance' seen in the gut and elsewhere. Certain regions of the brain, notably the floor of the third ventricle, also showed lesions of a more proliferative or granulomatous type. Finally, there was evidence of a peripheral neuropathy, affecting both motor and sensory fibres.

\section{DISCUSSION}

Apart from the neurological state in the terminal phase of the illness, and the pathological findings in the nervous system, this may be regarded as a typical 
case of Whipple's disease. Clinically, the case of Lampert et al. (1962) resembled ours in showing a severe paralysis of gaze, but differed in that the mental disturbance was more protracted and more profound, and in showing clonic movements of the limbs. Pathologically, our case, like those described by Sieracki et al. (1960) and Lampert et al. (1962), showed ependymal granulations, but appears to differ from them in the distribution of the 'Whipple substance' in the brain and spinal cord.

There is a clear resemblance between the lesions of the first type which we have described and those described by Lampert et al. They found randomly distributed clusters of microglial cells, containing P.A.S.- positive material throughout the grey matter of the brain and especially in the basal ganglia. They add: 'Frequently, small P.A.S.-positive granules were noted in apparently extracellular position, but it was felt that these granules lay within processes of microglial cells'. The disagreement here is probably one of the interpretation of the histological picture. Findings not corresponding to ours include a diffuse scattering of similar microglial cells in the white matter, and areas (not specified) of neurone loss and gliosis, which included heavy accumulations of abnormal microglial cells. In our case the white matter (except that of the fornix and cerebellum) contained no such lesions, and the only place showing nerve cell loss was the floor of the third ventricle, where the lesions were mainly of the second type.

How lesions of the first type arise is uncertain. It is plain, however, that the material has not simply diffused through the walls of capillaries, since it is not found in relation to vessels, and the capillary walls look entirely normal. Assuming that the blood-brain barrier was intact, it is reasonable to suppose that the substance, or its precursor, had been extracted from the blood by glial cells. It seems from electron microscope studies (e.g., Horstman and Meves, 1959; Luse and Harris, 1961) that there is no appreciable intercellular space in either grey or white matter, and that the 'background substance' of the brain consists of the processes of glial cells. If this is so, it is very likely that most of the apparently extracellular P.A.S.-positive material in our case is in fact contained in glial processes. Some, but not all, of the lesions include microglial phagocytes, which contain the material in concentrated form. We are inclined to regard these cells as scavengers, digesting particles of 'Whipple substance' liberated from glial processes, rather than as local factories engaged in elaborating the substance.

The fact that lesions of this type are practically confined to grey matter calls for an explanation. On this, again, we can only speculate. Since the nerve cells themselves appear to be unaffected, one is tempted to look for an explanation in terms of a difference between the biochemical activities of the satellite glial cells of the grey matter, which are believed to mediate the nutrition of nerve cells, and those of the oligodendrocytes of the white matter, which are supposedly concerned with the maintenance of myelinated axons. Whatever the answers to these questions may be, one thing seems clear: the substance is relatively harmless. This can be inferred from the lack of visible changes in nerve cells and processes, the absence of glial reaction, and, on the clinical side, from the lack of evidence of generalized cerebral disease.

Since we know of no other cerebral disorder in which lesions of this type and distribution are found, we are inclined to regard them as effects of Whipple's disease, and as evidence that in this condition there is an abnormal substance circulating in the blood. In regard to the second type of lesion, consisting of cellular proliferation in the hypothalamus and other localized areas, with phagocytic cuffing of small vessels, the situation is different. The clinical features of fluctuating ophthalmoplegia, unsteadiness, nystagmus, disorientation, and amnesia for recent events, combined with the findings of a peripheral neuropathy and cellular proliferation in the hypothalamus, all point to the conclusion that the patient was suffering from a form of Wernicke's encephalopathy.

It is now generally agreed (see e.g., Girard, Devic, and Garde, 1956; Pentschew, 1958; Meyer, 1958) that Wernicke's encephalopathy is primarily due to a deficiency in vitamin $B_{1}$ (thiamine). It occurs predominantly in chronic alcoholics, but is also a well-recognized complication of a number of gastrointestinal diseases, in which it is regarded as a result of defective or disordered assimilation of foodstuffs (Erbslöh, 1958). It is only too probable that our patient belonged to this latter group.

The essential lesions of Wernicke's encephalopathy consist in a proliferation of astrocytes, microglia, and capillaries, most marked in the tissues around the floor of the third ventricle, and to a lesser extent in the vicinity of the other parts of the ventricular system. The degeneration of nervous tissue which accompanies this is usually partial, and is held to be secondary to the vascular and cellular changes. The mammillary bodies are usually, but not always, the site of the most severe changes. Capillary haemorrhages sometimes occur, but probably as terminal events in advanced cases. In our case, the mammillary bodies are involved, but less so than the tuber cinereum. For the rest, minor lesions of this second type are found in a number of areas, all close to the ventricular system. It is only in the hypothalamus and ansa lenticularis that we find 
damage to nervous tissue, evidenced by loss of nerve cells and processes, and fibrillar gliosis. The fact that the perivascular phagocytic cells contain P.A.S.positive material can reasonably be attributed to a leaky state of the small vessels of these regions, such as is presumably present in haemorrhagic cases of Wernicke's encephalopathy, and to the presence of 'Whipple substance', or its precursor, in the blood. A minor piece of evidence that such a substance was present in the circulating blood, and was capable of leaving the circulation, is the finding of a layer of extracellular P.A.S.-positive material adhering to the free surface of the ependyma and in places to the surface of the choroid plexus.

Comparing this case with that of Lampert et al. (1962), it is hard to decide to what extent the lesions which they describe differ from ours. They found, as we did, scattered deposits of intra- and extracellular P.A.S.- positive material in the grey matter of the brain. Their general description of the lesions seems to include elements of both the main types of lesion observed by us; and although they do not enumerate the sites of the lesions in detail, there is some reason to suspect that in their case, as in ours, the disease was complicated by a form of Wernicke's encephalopathy.

Regarding the other types of lesion observed in this case, there is not much to be said. The third type, so-called granular ependymitis, is a nonspecific change which is seen in a number of disease conditions (Greenfield, 1958), or even in the absence of overt cerebral disease. The fourth type, consisting of patches of spongy degeneration, may also be non-specific; on the other hand, it is mentioned by Erbslöh (1958) as a frequent finding in cases of gastrointestinal disease complicated by Wernicke's encephalopathy. Finally, the appearance of peripheral neuropathy is fully compatible with a mild state of beri-beri.

In summary, we believe our findings can be interpreted as follows. As a result of Whipple's disease, the patient's blood contained an abnormal substance, traces of which passed into the cerebrospinal fluid, or were extracted from the blood by satellite glial cells. Towards the end of his life he developed a vitamin deficiency, which resulted in a peripheral neuropathy and a Wernicke's encephalopathy, in which the proliferated cerebral histiocytes took up the abnormal substance which had diffused through the walls of diseased vessels.

\section{SUMMARY}

A case of Whipple's disease, with neurological complications, is described and compared with other cases in the literature.

The pathological findings suggest that in this disease an abnormal substance is present in the blood, which may form scattered deposits in the brain, and in our case at least, that the picture is complicated by a form of Wernicke's encephalopathy.

\section{REFERENCES}

Casselman, W. G. B., Macrae, A. I., and Simmons, E. H. (1954) J. Path. Bact., 68, 67.

Erbslöh, F. (1958). In Handbuch der speziellen pathologischen Anatomie und Histologie, edited by $O$. Lubarsch, F. Henke, and R. Rössle, Band 13, Teil 2, Bandteil B, pp. 1699-1716. Springer, Berlin.

Farnan, P. (1958). J. clin. Path., 11, 382.

Girard, P. F. Devic, M., and Garde, A. (1956). Rev. neurol., 94, 493.

Greenfield, J. G. (1958). Neuropathology. Arnold, London.

Horstman, E., and Meves, H. (1959). Z. Zellforsch, 49, 569.

Lampert, P., Tom, M. I., and Cumings, J. N. (1962). Neurology, 12, ‡ 65.

Luse, S. A., and Harris, B. (1961). Arch. Neurol., 4, 129.

Meyer, A. (1958). In Neuropathology, edited by J. G. Greenfield, W. Blackwood, W. H. McMenemey, A. Meyer, and R. M. Norman. Arnold, London.

Pentschew, A. (1958). In Handbuch der speziellen pathologischen Anatomie und Histologie, edited by O. Lubarsch, F. Henke, and R. Rössle, Band 13, Teil 2, Bandteil B, pp. 2220-2234. Springer, Berlin.

Sieracki, J. C., Fine, G., Horn, R. C., and Bebin, J. (1960). J. Neuropath. exp. Neurol., 19, 70-75.

Whipple, G. H. (1907). Bull. Johns Hopk. Hosp., 18, 382. 